\title{
Bridging Sexual Boundaries: Men Who Have Sex with Men and Women in a Street-Based Sample in Los Angeles
}

\author{
Pamina M. Gorbach, Ryan Murphy, Robert E. Weiss, \\ Christopher Hucks-Ortiz, and Steven Shoptaw
}

\begin{abstract}
The purpose of the study was to determine the potential contribution of bisexual men to the spread of HIV in Los Angeles. We compare the characteristics and behaviors of men who have sex with men and women (MSMW) to men who have sex with only women (MSW) and men who have sex with only men (MSM) in Los Angeles. Men $(N=1,125)$ who participated in one of the two waves of data collection from 2005 to 2007 at the Los Angeles site for NIDA's Sexual Acquisition and Transmission of HIV-Cooperative Agreement Program were recruited using Respondent Driven Sampling. Participants completed Audio Computer Assisted Self Interviews and received oral HIV rapid testing with confirmatory blood test by Western Blot and provided urine specimens for detection of recent powder cocaine, crack cocaine, methamphetamine, or heroin use. MSM, MSW, or MSMW were defined by the gender of whom they reported sex with in the past 6 months. Chi-square tests and ANOVAs were used to test independence between these groups and demographic characteristics, substance use, and sexual behaviors. We fit generalized linear random intercept models to predict sexual risk behaviors at the partner level. Men were mostly of low income, unemployed, and minority, with many being homeless; $66 \%$ had been to jail or prison, $29 \%$ had ever injected drugs, and $25 \%$ had used methamphetamine in the past 30 days. The sample had high HIV prevalence: 12\% of MSMW, 65\% of MSM, and 4\% of MSW. MSMW were behaviorally between MSW and MSM, except that more MSMW practiced sex for trade (both receiving and giving), and more MSMW had partners who are drug users than MSW. Generalized linear random intercept models included a partner-level predictor with four partner groups: MSM, MSMW-male partners, MSMWfemale partners, and MSW. The following were significantly associated with unprotected anal intercourse (UAI): MSW (AOR 0.15, 95\% CI 0.08, 0.27), MSMW-female partners (AOR 0.4, 95\% CI 0.27, 0.61), HIV-positive partners (AOR 2.03, 95\% CI 1.31, 3.13), and being homeless (AOR 1.37, 95\% CI 1.01, 1.86). The factors associated with giving money or drugs for sex were MSMW-female partners (AOR 1.70, 95\% CI 1.09, 2.65), unknown HIV status partners (AOR 1.72, 95\% CI 1.29, 2.30), being older (AOR 1.02, 95\% CI 1.00, 1.04), history of incarceration (AOR 1.64, 95\% CI 1.17, 2.29), and being homeless (AOR 1.73, 95\% CI 1.27, 2.36). The following were associated with receiving money or drugs for sex: MSW (AOR 0.53,
\end{abstract}

Gorbach and Murphy are with the Department of Epidemiology, UCLA School of Public Health, Los Angeles, CA, USA; Gorbach is with the Division of Infectious Diseases, David Geffen School of Medicine at UCLA, Los Angeles, CA, USA; Weiss is with the Department of Biostatistics, UCLA School of Public Health, Los Angeles, CA, USA; Hucks-Ortiz and Shoptaw are with the Department of Family Medicine, David Geffen School of Medicine at UCLA, Los Angeles, CA, USA; Shoptaw is with the Department of Psychiatry and Biobehavioral Sciences, David Geffen School of Medicine at UCLA, Los Angeles, CA, USA.

Correspondence: Pamina M. Gorbach, BERG—Department of Epidemiology, University of California, Los Angeles-CHS 73-310, P. O. Box 957353, 10880 Wilshire Blvd., Suite 540, Los Angeles, CA 900957353, USA. (E-mail: pgorbach@ucla.edu) 
95\% CI 0.32, 0.89), African American (AOR 2.42, 95\% CI 1.56, 3.76), Hispanic (AOR 1.85, 95\% CI 1.12, 3.05), history of incarceration (AOR 1.44, 95\% CI 1.04, 2.01), history of injecting drugs (AOR 1.57, 95\% CI 1.13, 2.19), and had been recently homeless (AOR 2.14, 95\% CI 1.57, 2.94). While overall HIV-positive MSM had more UAI with partners of any HIV status than MSMW with either partner gender, among HIV-positive MSMW, more had UAI with HIV-negative and HIV status unknown female partners than male partners. Findings highlight the interconnectedness of sexual and drug networks in this sample of men-as most have partners who use drugs and they use drugs themselves. We find a concentration of risk that occurs particularly among impoverished minorities - where many men use drugs, trade sex, and have sex with either gender. Findings also suggest an embedded core group of drug-using MSMW who may not so much contribute to spreading the HIV epidemic to the general population, but driven by their pressing need for drugs and money, concentrate the epidemic among men and women like themselves who have few resources.

KEYWORDS Sexual bridging, MSMW, HIV risk behavior, HIV transmission risks

\section{INTRODUCTION}

Mathematical modeling of the transmission dynamics of sexually transmitted infections (STIs), including HIV, has shown that sexual mixing patterns within populations contribute to their maintenance and spread. ${ }^{1}$ Assortative sexual mixing (like-with-like) has been shown in such models to sustain STIs in distinct social groups. ${ }^{2}$ Assortative sexual mixing implies concordance between sex partners by factors such as age, residence location, ethnicity, socioeconomic status, or the rate of sex partner acquisition. In contrast, disassortative mixing implies discordance in characteristics of partners and has been shown empirically to allow spread of STI from groups with high STI prevalence (e.g., sex workers) to others within a population. ${ }^{3,4}$ Such discordance between sexual partners may represent a "bridge" between sexual networks because sexual networks are largely composed of individuals concordant for geographic residence, age, ethnicity, and sexual activity level. ${ }^{3,5,6}$ Such bridging enhances the movement of STI across networks when it exposes an individual from a low-risk network (one with few STIs and little risk behavior) to a person more likely to have an STI because they are from a high-risk network (one with more STIs and more risk behaviors).

The types of discordance between partner characteristics that have been associated with STIs includes discordance by number of lifetime partners, ${ }^{7,8}$ by race/ethnicity, ${ }^{3-5,9}$ and by age with HIV among men who have sex with men $(\mathrm{MSM})^{10}$ and in Sub-Saharan Africa also with HIV between young women and older male partners. ${ }^{11}$ The characteristic less studied for discordance is drug use and particularly drug discordance by gender of sexual partners as these partnerships may involve more than one type of discordance. Given that a significant number of sex partners of IDUs have been shown to be non-IDUs, ${ }^{12}$ non-injecting drug users are also likely to have many non-drug-using partners. Discordance by more than one characteristic may represent compounded risk for transmission across networks.

To establish and sustain a generalized epidemic in the USA, a large number of HIV transmissions from those with the highest HIV prevalence, MSM, to women would be required. Men who have sex with both men and women (MSMW) would serve as the bridge for HIV. Among women and MSMW, the majority of persons with new diagnoses of HIV/AIDS are African American. In addition, African 
American MSM report sex with women more frequently than do Hispanic or White MSM. ${ }^{13}$ This suggests that bridging among MSMW may involve more minority men and women.

As with the national epidemic, in Los Angeles County (LAC), the HIV epidemic has been concentrated among African Americans and Latinos; disproportionately, there are more recent infections and new diagnoses among African Americans and Latinos than Caucasians. ${ }^{14}$ Moreover, LAC's HIV epidemic from the beginning has involved primarily MSM and MSMW who together account for $76.1 \%$ of cases. Recent increases in the number of African American and Latina women diagnosed with HIV/AIDs raises questions as to whether the concentrated epidemic may be shifting to a generalized epidemic that involves more women, and specifically minority women. To determine the potential contribution of MSMW to the transmission of HIV from MSM to heterosexuals in Los Angeles, we assessed the amount and types of risk practiced by HIV-positive and HIV-negative men who were recruited using respondent driven sampling (RDS) in Los Angeles. We compare the characteristics and behaviors of MSMW to MSW and MSM.

\section{METHODS}

Participants were men who participated in one of the two waves of data collection (2005-2007) at the Los Angeles site for NIDA's Sexual Acquisition and Transmission of HIV-Cooperative Agreement Program. The sample for each wave was recruited using RDS, ${ }^{15}$ which is described in greater detail elsewhere in this volume. $^{26}$ Briefly, for each of the two waves of data collection, an initial set of "seeds" who were MSM or drug users was passively recruited via advertisements posted on walls and online. Each seed phoned a number, made an appointment, arrived at the recruitment site, completed all questionnaires, and provided all biological samples for total compensation of $\$ 50$ for this visit. As participants finished, they were provided with coupons to recruit people they knew who were MSM, a drug user, both MSM and a drug user, or whom were their sexual partner. The instructions for drug user coupons stated they should give a coupon to people "each of whom you think is a drug user who has used drugs like cocaine, methamphetamine, heroin, or crack (or who has injected some other drug) in the past six months...”. The instructions for the MSM coupon were similar. Sex partner coupons were also provided for individuals with whom they had had sexual intercourse with in the past 6 months. The seed received \$20 for each qualified individual enrolled in the study. Participants had to be 18 years of age or older and (1) MSM or MSMW who engaged in any anal intercourse (AI) in the past 6 months and/or (2) a man or woman who used powder cocaine, crack cocaine, heroin, or methamphetamine in the past 6 months; or recruited by one of the latter as a sexual partner or drug-using partner. Differences existed between waves 1 and 2 in the number of recruitment vouchers provided to participants. In wave 1, participants were offered up to seven coupons to recruit three people known to be either MSM and/or drug users, three coupons to recruit their sexual partners, and one coupon to either (a) a female sexual partner of an MSM or b) a non-drug-using sex partner of a drug user. The maximum possible compensation for study participation was $\$ 190$. In wave 2, participants were offered up to four coupons: two to recruit people known to be a MSM or drug user and two to recruit female sexual partners. After approximately six months of wave 2 , the number of coupons was increased to six because fewer coupons proved insufficient to sustain the recruitment chains. 
Participants completed Audio Computer Assisted Self Interviews that collected detailed information on the following: (1) demographic characteristics; (2) drug use (powder cocaine, crack cocaine, methamphetamine, or heroin); (3) sexual risk behaviors-number of male and female sexual partners in the prior 30 days and the past 6 months, number of specific behaviors engaged in over the past 6 months while having sex with male and with female partners; (4) specific sexual and drug use behaviors with each of up to six sexual partners (in wave 1 the last three sexual partners and a main or female partner but in wave 2 the language was changed to allow for any three partners and a main or female partner); and (5) characteristics and behaviors of each of those sex partners. In addition, all individuals underwent an oral HIV rapid test with confirmatory blood test by Western Blot for those testing positive and provided urine specimens for detection of recent powder cocaine, crack cocaine, methamphetamine, or heroin use. Individuals were also tested for sexually transmitted infections but these data are not included in this analysis.

For the main analyses, individuals were defined as MSM, MSW, or MSMW if they were men who reported sex in the past 6 months with only men, with only women, or with both. We used chi-square and ANOVA procedures to test differences between these groups and demographic and substance use variables. We analyzed behavior reported during the last sex act with each of three of sexual partners in last 6 months. We constructed a partner-level predictor with four groups: (1) MSM-all partners, (2) MSMW-male partners, (3) MSMW-female partners, and (4) MSW-all partners to compare characteristics of the MSMW partners by gender (Table 2 and Figure 1) to MSM partners and MSW partners. We fit generalized linear random intercept models using sexual behavior group and demographics as the independent variables and partner-level sexual risk behaviors as the dependent variables. The latter included unprotected anal intercourse (UAI), giving or getting drugs or money for sex (sex trade), having a partner from the same neighborhood,

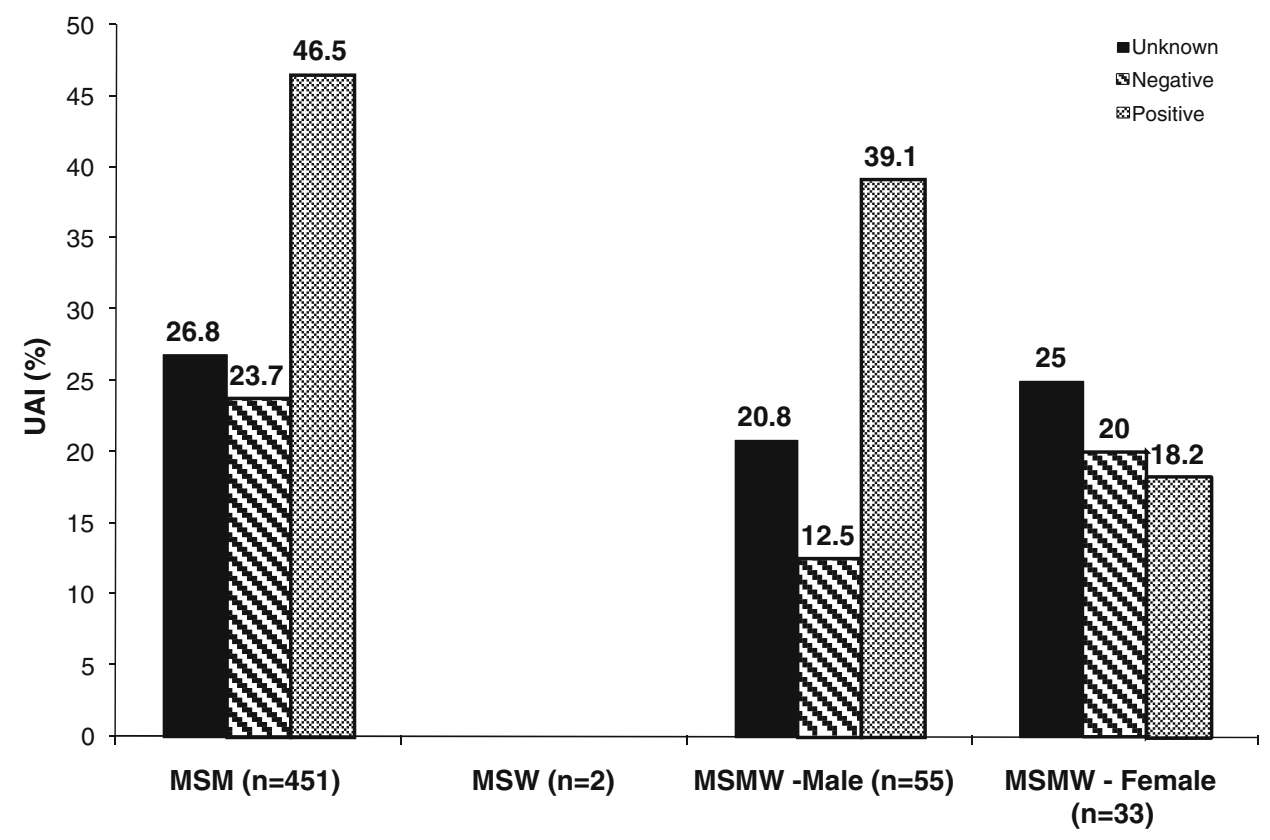

FIGURE 1. Unprotected anal intercourse by HIV status of partner among HIV+ males. 
disclosing one's HIV status to a partner, having a concurrent partner, and having a partner who has concurrent partners. Since only UAI and sex trade had significant covariates, multivariate models for these outcomes only are presented in Table 3. All models also included the following demographic characteristics and risk behaviors: age, race/ethnicity, HIV status, partner HIV status, history of incarceration, history of injection drug use, and homelessness. Because of changes between waves in the coupon distribution and language about sex partners described, wave was controlled for in all analysis. Analyses were fit using SAS (version 9.1) Proc Glimmix (SAS Institute, Cary, NC, USA).

All scientific and research procedures were overseen by the UCLA Human Subjects Protection Committee and the RAND Institutional Review Board.

\section{RESULTS}

Across both waves combined, there were 461 MSMW, 431 MSM, and 233 MSW. Data from each of up to three recent sexual partners was analyzed for these men producing 845 partners of MSMW, 806 partners of MSM, and 441 partners of MSW individual sex partners, respectively. Among the 845 partners reported by MSMW, gender of sex partner was reported for 349 male partners and 496 female partners. There was a high prevalence of HIV among the male participants compared to the general population of the USA-overall 31\%-with great variation between the groups. The highest was among MSM (64\%) followed by MSMW $(12 \%)$ and the lowest was among MSW (4.4\%). In general, the sample was composed of men who were of low income, minority, largely unemployed, homeless, and with a history of incarceration. More MSMW were also in the lowest categories of income and education and reported being homeless slightly more than MSM or MSW. Participants reported an average of 3.9-10.3 sexual partners in the last 6 months. MSMW reported the most sex partners although the difference was not statistically significant. Many men in all groups were drug users, and overall, about one third were injectors. There was variation in types of drugs used with MSW using more heroin and cocaine than the other men and MSM using more methamphetamines. The percent of MSMW reporting and testing positive for drug use fell in between that of MSM and MSW (Table 1).

MSMW and MSW shared many characteristics and are more similar to each other than to MSM. Differences by race were greatest; about twice as many of the MSMW (and MSW) were African American compared to MSM. Most MSW and MSMW were also US born, unlike MSM of whom about one fourth were born outside the USA. More MSMW with female partners reported having concurrent partners than men from the other two groups. There were no significant differences in alcohol use (data not shown), and although more MSM used methamphetamines, more MSMW reported injecting methamphetamine in the past month (although not significantly) (Table 1).

Overall, about a third of men reported a main or primary partner. Fewer of the MSMW's male partners were reported as main partners compared to MSM or MSW; more MSW's partners were main. More than one-half of MSW and MSMW's partners were African American, which also was the case for both male and female partners of MSMW. Fewer of the male partners for MSMW and MSM were from their own neighborhoods compared to MSW. Mean ages of partners were within 10 years of participant ages across all groups, suggesting little age mixing. MSMW reported more injecting partners than the other groups. Moreover, injecting 


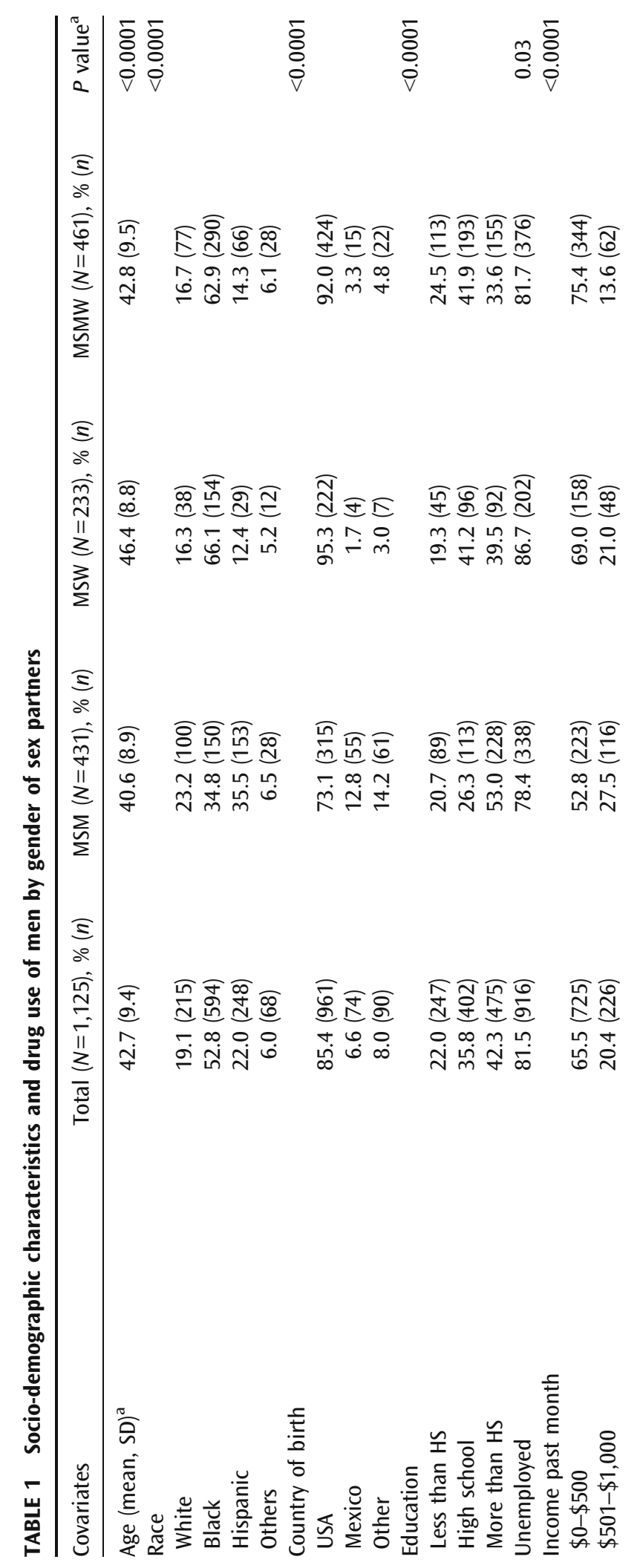




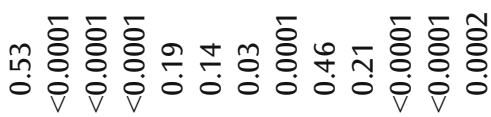

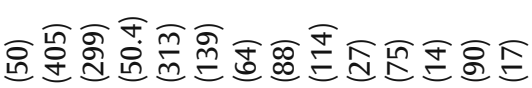

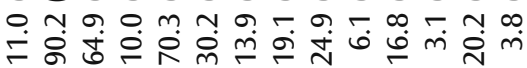

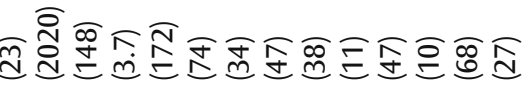

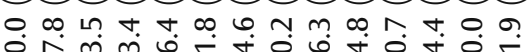

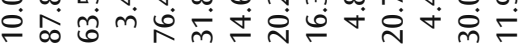

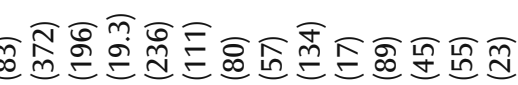

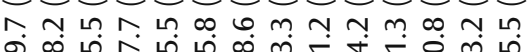

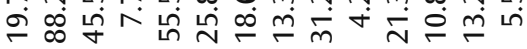

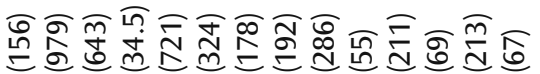

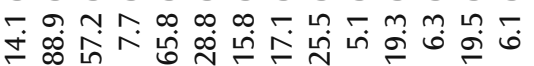

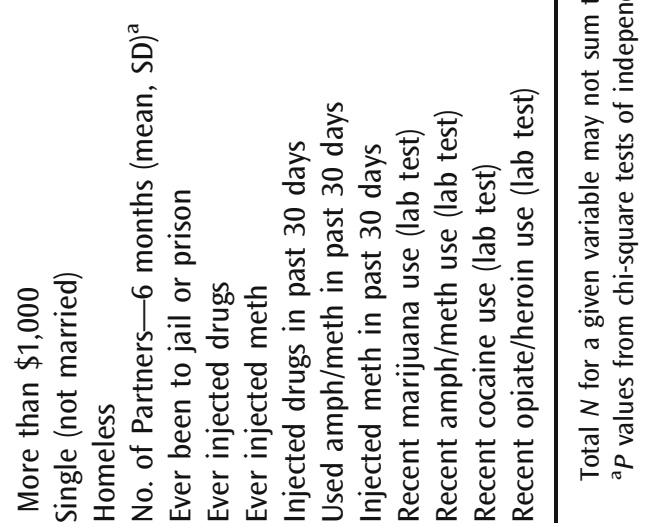


partners were reported by more MSMW with male partners than either MSMW with female partners or any of the men reported of their partners. Sex while high or with a partner who was high was reported by more MSMWs with female partners than by other men yet more MSW reported a partner who was high on drugs during sex than reported by men with any other type of partner. Interestingly, MSM were the least likely to report giving a partner money or drugs for sex and more MSMW reported paying female partners for sex than the other groups. Almost twice as many MSMW reported receiving money or drugs for sex with either male or female partners than MSM or MSW (Table 2).

MSMW reported more sexual partners over the last six months than either MSM or MSW and they reported more male than female partners. Most unprotected sex (i.e., sex without using condoms) was reported with female partners but the most UAI was reported by MSM and MSMW with their male partners. Among HIV-positive men, most UAI was with HIV-positive partners except among the MSMW, many fewer of whom reported UAI with a HIV-positive female partner (Figure 1). While, overall, more HIV-positive MSM reported UAI with partners of any HIV status than MSMW with either partner gender, more HIV-positive MSMW reported UAI with HIV-negative and HIV status unknown female partners than male partners.

Multivariate models assessed associations between behaviors with up to six recent sexual partners (Table 3). The following were significantly associated with being less likely to report UAI: being a MSW or a MSMW with female partners compared to a MSM. Those more likely to report UAI reported HIV-positive partners and being homeless. The model demonstrates that the following had higher odds of reporting giving money or drugs for sex: MSMW with female partners, men with partners of unknown HIV status, are older, with a history of incarceration, and who are homeless. Finally, receiving money or drugs for sex was associated with higher odds of being African American, having a history of incarceration, a history of injecting drugs, and being homeless, while MSW had significantly lower odds of having traded sex for money or drugs than MSM.

\section{DISCUSSION}

This unique sample of men recruited by their peers provides insight into how drugs and sex weave into the relationships of men on the streets in the large, diverse city of Los Angeles. Our sample is unusual because it captured many poor, homeless men who use drugs and in doing so, also mix sexually with those of both genders. Almost half our sample were men who have sex with men and women (MSMW) in the past six months-a very high proportion of the sample given that in the US general population only about $1 \%$ of men $15-44$ years of age have had both male and female sexual partners in the last 12 months. ${ }^{16}$ This enables us to describe the behavior of these men that are often difficult to capture using more standard methods of sampling. Moreover, our sample contained partner specific data on recent partners, enabling us to analyze within men differences in behavior by the gender of their partners. The men in our sample shared many demographic and behavioral characteristics regardless of the gender of their sex partners, but there were important distinctions in the contexts in which sex occurred. It is where and how the sexual networks of these men mix that could account for spread of HIV or alternatively entrench it within certain subgroups. 


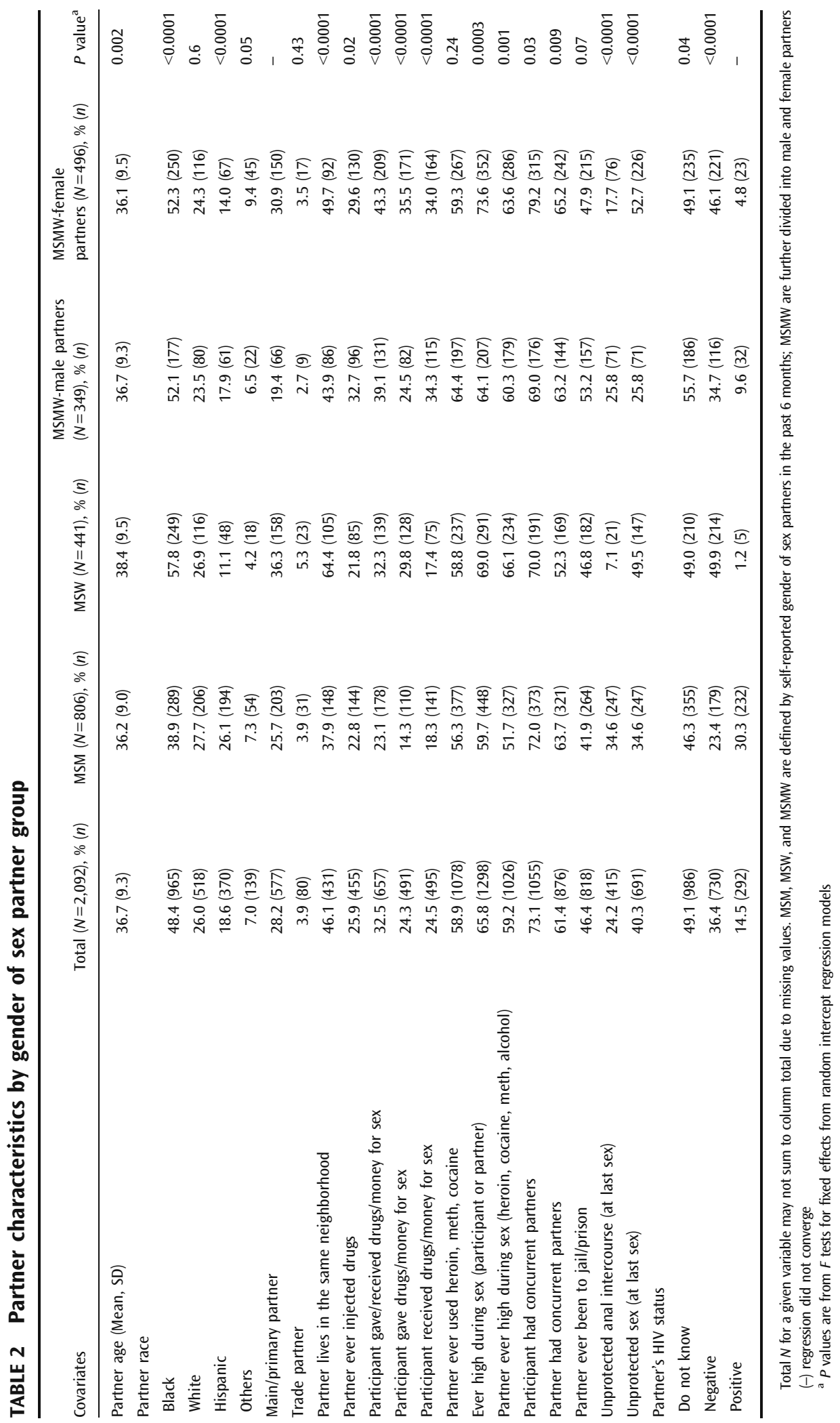


TABLE 3 Random intercept regression models for unprotected anal sex (UAI), giving or getting money or drugs for sex

\begin{tabular}{|c|c|c|c|c|c|}
\hline Dependent variable & Covariate & OR & $\mathrm{Cl}$-lower & $\mathrm{Cl}$-upper & $P$ value \\
\hline \multirow[t]{15}{*}{ UAI } & Intercept & 0.56 & 0.25 & 1.25 & 0.16 \\
\hline & MSW & 0.15 & 0.08 & 0.27 & $<0.001^{*}$ \\
\hline & MSMW-male & 0.69 & 0.45 & 1.05 & 0.08 \\
\hline & MSMW-female & 0.40 & 0.27 & 0.61 & $<0.001^{*}$ \\
\hline & $\mathrm{HIV}+$ & 0.77 & 0.53 & 1.14 & 0.19 \\
\hline & Partner HIV+ & 2.03 & 1.31 & 3.13 & $0.001^{*}$ \\
\hline & Partner HIV unknown & 0.99 & 0.72 & 1.35 & 0.94 \\
\hline & Age & 0.99 & 0.97 & 1.00 & 0.10 \\
\hline & Black & 0.91 & 0.62 & 1.36 & 0.66 \\
\hline & Hispanic & 1.12 & 0.73 & 1.72 & 0.59 \\
\hline & Other-race & 0.85 & 0.44 & 1.64 & 0.63 \\
\hline & Been to jail (ever) & 1.27 & 0.93 & 1.74 & 0.13 \\
\hline & Injected drugs & 1.24 & 0.90 & 1.71 & 0.18 \\
\hline & Homeless (past year) & 1.37 & 1.01 & 1.86 & $0.04^{*}$ \\
\hline & Wave 2 & 1.21 & 0.91 & 1.62 & 0.20 \\
\hline \multirow{15}{*}{$\begin{array}{l}\text { Give money/drugs } \\
\quad \text { for sex }\end{array}$} & Intercept & 0.03 & 0.01 & 0.06 & $<0.001^{*}$ \\
\hline & MSW & 1.11 & 0.68 & 1.82 & 0.68 \\
\hline & MSMW-male & 0.99 & 0.62 & 1.59 & 0.97 \\
\hline & MSMW-female & 1.70 & 1.09 & 2.65 & $0.02^{*}$ \\
\hline & $\mathrm{HIV}+$ & 0.74 & 0.47 & 1.16 & 0.18 \\
\hline & Partner HIV+ & 0.81 & 0.46 & 1.40 & 0.45 \\
\hline & Partner HIV unknown & 1.72 & 1.29 & 2.30 & $<0.001^{*}$ \\
\hline & Age & 1.02 & 1.00 & 1.04 & $0.02 *$ \\
\hline & Black & 1.99 & 1.29 & 3.08 & $0.002^{*}$ \\
\hline & Hispanic & 1.16 & 0.69 & 1.94 & 0.57 \\
\hline & Other-race & 1.74 & 0.88 & 3.45 & 0.11 \\
\hline & Been to jail (ever) & 1.64 & 1.17 & 2.29 & $0.004^{*}$ \\
\hline & Injected drugs & 1.12 & 0.80 & 1.56 & 0.51 \\
\hline & Homeless (past year) & 1.73 & 1.27 & 2.36 & $<0.001^{*}$ \\
\hline & Wave 2 & 1.15 & 0.85 & 1.54 & 0.36 \\
\hline \multirow{15}{*}{$\begin{array}{l}\text { Get money/drugs } \\
\text { for sex }\end{array}$} & Intercept & 0.07 & 0.03 & 0.17 & $<0.001^{*}$ \\
\hline & MSW & 0.53 & 0.32 & 0.89 & $0.02^{*}$ \\
\hline & MSMW-male & 1.43 & 0.91 & 2.23 & 0.12 \\
\hline & MSMW-female & 1.31 & 0.85 & 2.02 & 0.23 \\
\hline & HIV+ & 0.79 & 0.51 & 1.21 & 0.28 \\
\hline & Partner HIV+ & 0.86 & 0.52 & 1.43 & 0.55 \\
\hline & Partner HIV unknown & 1.31 & 0.98 & 1.75 & 0.07 \\
\hline & Age & 1.00 & 0.98 & 1.02 & 0.86 \\
\hline & Black & 2.42 & 1.56 & 3.76 & $<0.001^{*}$ \\
\hline & Hispanic & 1.85 & 1.12 & 3.05 & $0.02 *$ \\
\hline & Other-race & 1.49 & 0.74 & 3.00 & 0.27 \\
\hline & Been to jail (ever) & 1.44 & 1.04 & 2.01 & $0.03 *$ \\
\hline & Injected drugs & 1.57 & 1.13 & 2.19 & $0.007^{*}$ \\
\hline & Homeless (past year) & 2.14 & 1.57 & 2.94 & $<0.001^{*}$ \\
\hline & Wave 2 & 0.96 & 0.72 & 1.29 & 0.79 \\
\hline
\end{tabular}


MSMW have been shown to reside behaviorally between MSM and MSW for key risk behaviors such as numbers of sexual partners and frequency of sex. ${ }^{17}$ In our sample, MSMW also occupied the middle on most risk variables, yet what distinguished them from the other men is that about twice as many reported receiving drugs or money for sex as MSM or MSW. More of the MSMW we studied were also in the lowest income and education categories and more were homeless than either MSM or MSW, suggesting that the extreme poverty and social marginalization interact with these men's lives to drive commercial sex activitiesto facilitate either survival or desired sexual expression. Our multivariate analysis further illustrates that those reporting receipt of drugs or money for sex were more likely to report histories of incarceration and of injection drug use, further emphasizing associations between social marginalization and trading of sex. Finally, men who reported being paid for sex were more likely to be African American, findings consistent with a qualitative study of African American MSM in Los Angeles in which exchange of sex or drugs, money, or basic needs were reported to be common. ${ }^{18}$

The frequent experiences of incarceration and homelessness in this sample imply that these men spend much time in jails and on the streets-places at which sexual networks can be connected. Such locales, however, have barriers that affect the practice of safe sex. For example, African American men in Los Angeles have reported a lack of availability of condoms in jails and homeless shelters as a barrier to protected sex. ${ }^{18}$ This suggests that when in jail or on the streets, those who may not normally practice risky behavior are faced with structural barriers to being safe and therefore engage greater risk in these settings.

The MSMW in our study appear to be sexually bridging geographic as well as gender networks. Many MSM and about one-half MSMW-male partners reported sexual partners from other neighborhoods, which contrasts with MSW and the MSMW-female partners, many of whom reported partners from their own neighborhood. For these MSM and MSMW, concerns about same-sex behaviors being found out by acquaintances, friends, or family members may contribute to looking outside one's social network to encounter male sex partners. There would be few similar concerns when seeking female partners, as most of these were reported from the men's neighborhoods. Such neighborhood differences suggest that heterosexual networks may be more localized, whereas sexual networks of MSM and MSMW may be more far reaching. This suggests more disassortative mixing by location among men with male partners than with female partners. As such, MSMW can function as potential bridges for HIV to cross, as the men travel for some sexual partners while maintaining others in their own neighborhood, thereby connecting two distinct neighborhoods. We also observed in our data high rates of both dissasortative mixing and concurrent sexual partnerships, which enhances the efficiency of HIV spread throughout populations.

In this sample, potential for transmitting HIV from MSMW to either men or women results largely from a lower use of condoms and because MSMW are having sex with both a very high HIV prevalence (MSM) and low HIV prevalence group (women). We found that the MSMW did report relatively little condom use with female partners, especially during vaginal intercourse, but many more reported condom use during sex with men. Other studies have shown minority MSMW use condoms as much as, if not more than MSMW of other races/ethnicities. ${ }^{19,20}$ Furthermore, African American and "other" race men who have both male and female partners tend to have less UAI with males than those men who have only 
male partners. However, MSMW who do not disclose their same-sex behaviors report more unprotected intercourse with female partners than those who do disclose. ${ }^{13}$ What is of concern in our study is that when we examine the practice of UAI among HIV-positive men (Figure 1), we observed MSMW reporting significant UAI with females partners of HIV-negative and of unknown HIV status. Given that UAI is the highest transmitting sexual risk behavior, possible transmission to these female partners is of great concern. This finding is surprising because the other HIV-positive men in the sample appear to "serosorting" by having UAI mostly with HIV-positive partners. Nevertheless, substantial proportions of the HIV-positive men in all groups report UAI with HIV negative and status unknown partner, demonstrating that risks for transmission is occurring between men as well as between men and women.

MSMW were predominantly African American in this sample, and most of the partners also were African American. This suggests a highly assortative sexual networking pattern that describes MSMW as a "core group" who largely mix with other minority men and women. The higher numbers of sexual partners, especially male partners, reported by MSMW compared to either MSM or MSW also confirms that these MSMW may function like a core group. A core group is defined as a group of individuals characterized by high rates of partner change (often with each other), longer duration of infection often related to poor access to health care, and highly efficient transmission of infection per exposure, all contributing to high rates of STIs. ${ }^{21}$ While such a dynamic has been noted before among African American MSM and been suggested to be a contributing factor to their higher prevalence of $\mathrm{HIV}^{22}$ it has also been shown that these men are also likely to be have disassortative mixing with regard to age, with many partnerships that have a difference of 10 years of age or greater. ${ }^{23}$ In our sample, we observe such assortative mixing by race/ ethnicity among African American participants; however, we see no evidence of pronounced disassortative age mixing. We do see evidence of mixing by HIV status, and this is a great cause for concern.

Drug use is an important dynamic that pervades the sexual choices of the men in this study. While MSMW reported methamphetamine use rates between MSM and MSW, they were more likely to have recently injected methamphetamine than even MSM. Clearly, when sex occurs in the context of drug use, it is usually higher risk. For example, IDU MSM are more likely than non-IDU MSM to report unprotected receptive anal intercourse (UAI) with casual partners, which was found to be strongly associated with HIV seroconversion in a prospective analysis of a cohort of MSM. $^{24}$ In our study, we also show that UAI was associated with a history of injecting drug use, suggesting the ability to practice safe sex is a particular challenge for drug users.

Using RDS produced a sample of very poor, minority, drug-using men; very few men were employed, well-educated, White, or young. This limits the generalizability of our findings to other MSMW in Los Angeles. Patterns of risk may be very different for MSMW that are not as marginalized as those in our sample. Another limitation is that it was beyond the scope of this manuscript for us to examine differences in sexual position among MSMW, a factor that affects the transmission probability. Future analyses of this dataset will address these research questions and shed further light on the potential contributions for transmission and dynamics of that risk among the men in our sample.

This study provides a rare look into the interconnectedness of sexual and drug behaviors in this sample of men-most of whom have sex partners who use drugs. 
The findings raise a flag of concern for the HIV-negative female sexual partners of MSMW who we show to be at considerable risk for HIV acquisition because of the proportion of HIV-positive MSMW who report UAI with them. Such risky sexual behavior likely occurs in the context of drug use or commercial sex, as we also found many MSMW reporting trading money or drugs for sex with female partners. What is borne out is that a concentration of risk occurs among impoverished minorities-where men, many of whom are HIV positive, commonly use drugs, trade sex, and have sex with either gender. These data provide support for the hypothesis that such "compound risk" enhances the frequency and variety of exposures, increasing the cumulative probability of transmission and, therefore, plays a major role in maintaining the endemicity of HIV in the settings where it occurs. $^{25}$ Our findings suggest an embedded core group of minority drug-using MSMW who may not so much contribute to spreading the HIV epidemic to the general population, but driven by their pressing need for drugs and money, may concentrate the epidemic among men and women like themselves who have few resources. What remains to be found is whether such dynamics also hold in younger groups of urban MSMW.

\section{ACKNOWLEDGMENT}

Drs. Shoptaw and Gorbach gratefully acknowledge the support of NIDA grant U01DA17394 for this work.

OPEN ACCESS This article is distributed under the terms of the Creative Commons Attribution Noncommercial License which permits any noncommercial use, distribution, and reproduction in any medium, provided the original author(s) and source are credited.

\section{REFERENCES}

1. Aral SO. Patterns of sexual mixing: mechanisms for or limits to the spread of STIs? Sex Transm Infect. 2000; 76(6): 415-416.

2. Anderson RM. Transmission dynamics of sexually transmitted infections. In: Holmes KK, et al, ed. Sexually Transmitted Diseases. New York: McGraw-Hill; 1999: 25-38.

3. Rothenberg RB, Potterat JJ. Temporal and social aspects of gonorrhea transmission: the force of infectivity. Sex Trans Dis. 1987; 15: 88-92.

4. Potterat JJ, Muth SQ, Rothenberg RB, et al. Sexual network structure as an indicator of epidemic phase. Sex Trans Infect. 2002; 78: i152-i158.

5. Laumann EO, Youm Y. Racial/Ethnic Group difference in the prevalence of sexually transmitted diseases in the United States: a network explanation. Sex Trans Dis. 1999; 26 (5): 250-261.

6. Laumann EO, Gagnon JH, Michael RT, et al. The Social Organization of Sexuality. Chicago: University of Chicago Press; 1994.

7. Gorbach PM, Drumright LN, Holmes KK. Discord, discordance \& concurrency: comparing individual and partnership-level analyses of new partnerships of young adults at risk of STI. Sex Transm Dis. 2005; 32(1): 7-12.

8. Drumright LN, Gorbach PM, Holmes KK. Do people really know their sexual partners?: concurrency, knowledge of partner behavior and sexually transmitted infections within partnerships. Sex Transm Dis. 2004; 31: 437-442.

9. Ford K, Sohn W, Lepkowski JM. Characteristics of adolescent's sexual partners and their association with use of condoms and other contraceptive methods. Fam Plann Perspect. 2001; 33: 100-105. 
10. Service S, Blower SM. HIV transmission in sexual networks: an empirical analysis. Proc $R$ Soc Lond B Biol Sci. 1995; 260(1359): 237-244.

11. Gregson SNC, Garnett GP, Mason PR, et al. Sexual mixing patterns and sex-differentials in teenage exposure to HIV infection in rural Zimbabwe. Lancet 2002; 359(9321): 18961903.

12. Howard DL, Latkin CA. A bridge over troubled waters: Factors associated with noninjection drug users having injection drug-using sex partners. J Acquir Immune Defic Syndr 2006; 42: 325-330.

13. McKirnan DJ, Stokes JP, Doll LS, et al. Bisexually active men: social characteristics and sexual behavior. J Sex Res. 1995; 32(1): 65-76.

14. HIV Epidemiology Program and L.A.C.D.o.P. Health, HIV/AIDS Semi-Annual Surveillance Summary. Los Angeles; 2008: 1-30.

15. Heckathorn DD. Respondent-driven sampling: a new approach to the study of hidden populations. Soc Probl. 1997; 44: 174-199.

16. Mosher WD, Chandra A, Jones J. Sexual behavior and selected health measures: men and women 15-44 years of age, United States, 2002. Adv Data. 2005; 362: 1-55.

17. Mercer $\mathrm{CH}$, Hart GJ, Johnson AM, et al. Behaviourally bisexual men as a bridge population for HIV and sexually transmitted infections? Evidence from a national probability survey. Int J STD AIDS. 2009; 20: 87-94.

18. Harawa NT, Williams JK, Ramamurthi HC, et al. Perceptions towards condom use, sexual activity, and HIV disclosure among HIV-positive African American men who have sex with men: implications for heterosexual transmission. J Urban Health. 2006; 83(4): 682-694.

19. Harawa NT, Greenland S, Bingham TA, et al. Associations of race/ethnicity with HIV prevalence and HIV-related behaviors among young men who have sex with men in 7 urban centers in the United States. J Acquir Immune Defic Syndr Human Retrovirol. 2004; 35: 526-536.

20. Bingham TA, Harawa NT, Johnson DF, et al. The effect of partner characteristics on HIV infection among African American men who have sex with men in the Young Men's Survey, Los Angeles, 1999-2000. AIDS Educ Prev. 2003; 15(1 Suppl A): 39-52.

21. Aral SO, Holmes KK, Padian NS, et al. Overview: individual and population approaches to the epidemiology and prevention of sexually transmitted diseases and human immunodeficiency virus infection. J Infect Dis. 1996; 174(Suppl 2): S127-S133.

22. Millett G, Flores SA, Peterson JL, et al. Explaining disparities in HIV infection among Black and White men who have sex with men: a meta-analysis of HIV-risk behaviors. AIDS. 2007; 21(15): 2083-2091.

23. Berry M, Raymond HF, McFarland W. Same race and older partner selection may explain higher HIV prevalence among black men who have sex with men. AIDS. 2007; 21(17): 2349-2350.

24. O'Connell JM, Lampinen TM, Weber AE, et al. Sexual risk profile of young men in Vancouver, British Columbia, who have sex with men and inject drugs. AIDS Behav. 2004; 8: 17-23.

25. Rothenberg R. Maintenance of endemicity in urban environments: a hypothesis linking risk, network structure and geography. Sex Transm Infect. 2007; 83(1): 10-15.

26. Iguchi M, Ober A, Berry S, Fain T, Heckathorn D. Simultaneous recruitment of drug users and men who have sex with men in the United States and Russia using respondent driven sampling: sampling methods and implications. J Urban Health. 2009; doi:10.1007/s11524-009-9365-4. 